\title{
Effect of charge on point defect size misfits from ab initio: Aliovalently doped $\mathrm{SrTiO}_{3}$
}

\author{
Hyojung Kim, Dallas R. Trinkle* \\ Department of Materials Science and Engineering, University of Illinois at \\ Urbana-Champaign, Urbana, IL 61801
}

\begin{abstract}
We demonstrate a computationally efficient method to calculate size misfits of point defects in ailovalently doped $\mathrm{SrTiO}_{3}$ using density functional theory. The size misfits of neutral and charged single point defects are calculated and linearly combined to estimate the effective size misfits of multiple point defects in aliovalently doped $\mathrm{SrTiO}_{3}$. The charge state has a demonstrable effect on point defect size misfits for both isolated and multiple defects. We attribute this to the different charge localization between the defective systems. The extra electron or hole for the charged isolated point defects compensates the difference of charge localization between the neutral point defects and aliovalently doped system. The sum of size misfits of charged isolated point defects accurately estimates size misfits for aliovalently doped $\mathrm{SrTiO}_{3}$, without charge correction which was possibly required due to the additional charge state of point defect. Keywords: size misfits, charged point defects, density functional theory, $\mathrm{SrTiO}_{3}$
\end{abstract}

\section{Introduction}

The perovskite material strontium titanate $\left(\mathrm{SrTiO}_{3}\right)$ is used in a wide range of applications including buffer layers for epitaxial thin films, microwave resonators, and catalysts [1, 2, 3]. The dielectric properties of $\mathrm{SrTiO}_{3}$ depend

\footnotetext{
* Corresponding author

Email address: dtrinkle@illinois.edu (Dallas R. Trinkle)
}

Preprint submitted to Journal of Computational Materials Science

February 14, 2016 
on its structural parameters such as lattice constants and the tolerance factor [4. Many theoretical and empirical models predict the structural parameters based on the composition of perovskites [5, 6, 7]. However, these models do not account for the effects of dopants or vacancies on the structural parameters, despite the fact that $\mathrm{SrTiO}_{3}$ is commonly doped by metals or trivalent rare earth elements to improve the dielectric properties [8]. Recently, Ubic et al. 9] controlled the $\mathrm{Sr}$ vacancy concentration in $\mathrm{SrTiO}_{3}$ by doping with the trivalent atoms ( $\mathrm{La}, \mathrm{Ce}$, and $\mathrm{Nd}$ ), and measured the lattice constants according to the vacancy concentration. The authors suggest an improved empirical model to evaluate the pseudo-cubic lattice parameter and tolerance factor of aliovalently doped $\mathrm{SrTiO}_{3}$ by accounting for the effective size of a vacancy at a $\mathrm{Sr}$ site.

Calculating formation volumes and energies of charged point defects using density functional theory (DFT) requires a careful approach to account for electrostatic interactions within the periodic cell and the potential shift [10, 11, 12]. In silicon, Bruneval et al. [13] described the potential bias caused by the charge state of point defects that significantly changes the formation volume and requires correction. They found that the corrected formation volume weakly depends on the defect type and charge state. Density functional theory studies of perovskites studies have focused on charged or neutral oxygen vacancies and their formation energies or electronic properties [14, 15, but not on formation volumes. The formation energy calculations for charged point defects require a charge correction to account for the potential shift in perovskites as well. These results suggest that the charge state could affect formation volume, and aliovalently-doped $\mathrm{SrTiO}_{3}$ with experimentally measured lattice constants [9] provides an ideal system to answer this question. Aliovalently-doped $\mathrm{SrTiO}_{3}$ contains two substitutional atoms $\left(\mathrm{Ln}_{\mathrm{Sr}}, \mathrm{Ln}=\mathrm{La}, \mathrm{Nd}, \mathrm{Ce}\right)$ with a single vacancy $\left(V_{\mathrm{Sr}}\right)$ at $\mathrm{Sr}$ atomic sites given by

$$
\mathrm{Ln}_{2} \mathrm{O}_{3} \stackrel{\mathrm{SrTiO}_{3}}{\rightarrow} 2 \mathrm{Ln}_{\mathrm{Sr}}^{\bullet}+V_{\mathrm{Sr}}^{\prime \prime}+3 \mathrm{O}_{\mathrm{O}}^{\times}
$$

These trivalent substitutional elements have a +1 charge, compensated by the -2 charge of the Sr vacancy. Shanthi and Sarma computed charge distributions 
in perfect $\mathrm{SrTiO}_{3}$ and in neutral $\mathrm{SrTiO}_{3}$ with a substitutional La solute on the Sr sublattice [16]. They find that the La does not yield midgap states, which is attributed to the La-derived electronic states occurring far below the Fermi energy. However, they didn't investigate the redistribution of charge in systems with multiple defects - i.e., aliovalently doped $\mathrm{SrTiO}_{3}$ - or the effect of charge transfer on point defect size misfits.

We demonstrate a computationally efficient method to calculate the effective size misfits of point defects in aliovalently doped $\mathrm{SrTiO}_{3}$ by linearly combining the size misfits of charged single point defects, without the need for charge correction. The size misfit calculation of single point defects produces a smaller finite size effect compared to the multiple defective geometry. We calculate the size misfits for multiple defects in aliovalently doped $\mathrm{SrTiO}_{3}$ and single point defects for different charge states. The sum of size misfits of single charged defects without correction matches well with the size misfits of neutral defect complexes, while the sum of size misfits of single neutral defects disagrees. In addition, electrons and holes have different sizes in Sr vacancies compared with substitutional sites. We attribute the disagreement in size misfits to differences in charge transfer for neutral point defects compared with defect complexes in aliovalently doped $\mathrm{SrTiO}_{3}$.

\section{Computational details}

We perform DFT calculations using the Vienna ab-initio simulation package (VASP) [17, 18 based on plane wave basis sets. The projector-augmented wave (PAW) potentials generated by Kresse [19] describe the $\mathrm{Sr}\left([\mathrm{Ni}] 4 s^{2} 4 p^{6} 5 s^{2}\right)$, Ti ([Ne] $\left.3 p^{6} 3 d^{2} 4 s^{2}\right), \mathrm{O}\left([\mathrm{He}] 2 s^{2} 2 p^{4}\right)$ and substitutional atoms (La, Ce, and $\mathrm{Nd}$ ) with the same core electron configuration $[\mathrm{Kr}]$. The Perdew-Burke-Ernzerhof (PBE) generalized gradient approximation (GGA) functional [20] accounts for electron exchange-correlation energy. A plane-wave energy cutoff of $850 \mathrm{eV}$ is required for an energy convergence of less than $1 \mathrm{meV} /$ atom. A $2 \times 2 \times 2$ Monkhorst-Pack [21] $k$-point grid samples the Brillouin zone of the $3 \times 3 \times 3 \mathrm{SrTiO}_{3}$ supercells (135 
atoms). Gaussian smearing is employed for integrating the density of states with a smearing width of $0.05 \mathrm{eV}$. All the atoms in the supercells are relaxed until the force on each atom is less than $5 \mathrm{meV} / \AA$. The $\mathrm{SrTiO}_{3}$ unit cell has a cubic structure with computed lattice constant $a=3.94 \AA$ and bulk modulus $B=$ $171.8 \mathrm{GPa}$, which agree well with the experimental values of $a=3.905 \AA[9]$ and $B=174 \mathrm{GPa}[22]$. It is assumed that the presence of defect does not change the bulk modulus significantly, thus negligible. We applied strain on unit cell from $\pm 0.1 \%$ to $\pm 0.4 \%$ by $0.1 \%$ difference to appropriate directions and relaxed ions with fixed volume to calculate three independent elastic constants $\mathrm{C}_{11}, \mathrm{C}_{12}$ and $\mathrm{C}_{44}$, where the calculated values are 320.3, 99.0 and 109 GPa respectively. Then, the bulk modulus $B$ is calculated by $1 / B=\left(\mathrm{S}_{11}+\mathrm{S}_{22}+\mathrm{S}_{33}\right)+2 *\left(\mathrm{~S}_{12}+\mathrm{S}_{13}+\mathrm{S}_{23}\right)$, where $\mathrm{S}_{i j}$ is the compliance tensor in Voigt notation which is the inverse of stiffness tensor. The calculated bulk modulus of $\mathrm{SrTiO}_{3}$ using various exchange correlation functionals is compared [23], showing the range of bulk modulus from 159 to $222 \mathrm{GPa}$ with different estimation of lattice constants. The electron density on each atomic site in the $\mathrm{SrTiO}_{3}$ supercells is calculated using the Bader charge integration method [24].

The size misfit of a point defect is the fractional change of lattice constant $a$ with respect to the concentration $c$ of the defect. We compute size misfits $\varepsilon_{b}$ by introducing point defects into a supercell and relaxing the atoms at fixed volume, which is found to be more suitable choice than the constant pressure method [10]. Point defects induce stress $\sigma$ in the cell, which yields the size misfits as 25,10 ]

$$
\varepsilon_{b}=\left.\frac{1}{a} \frac{d a}{d c}\right|_{c=0} \approx \frac{N \sigma}{3 B},
$$

where $N$ is the number of atoms in the supercell and $B$ is the bulk modulus of undefective $\mathrm{SrTiO}_{3}$. The size misfits of point defects in the aliovalently doped system are computed for $2 \times 2 \times 2$ and $3 \times 3 \times 3$ supercells to estimate finite size effects. The absolute errors for size misfit values between $2 \times 2 \times 2$ supercells and $3 \times 3 \times 3$ supercell are 0.010 and 0.038 for La and Ce, respectively. However, the Nd $2 \times 2 \times 2$ supercell calculations suffered from severe charge-sloshing 
exacerbated by finite size effects. All reported size misfits are from the $3 \times 3 \times 3$ supercells.

\section{Effective size misfits}

Figure 1 shows the geometries used to calculate the size misfits of point defects in aliovalently doped systems using a direct method and an indirect method. The direct method calculates the size misfits using the $\mathrm{SrTiO}_{3}$ structure containing two substitutional atoms and a Sr vacancy. In the direct method, the point defects are separated as far as possible from each other within the periodic geometry to minimize the effect of defect interactions on the size misfits. The absolute error of size misfits computed from two different atomic arrangements (i.e., different distances between point defects) using the $2 \times 2 \times 2$ supercell are 0.001 and 0.015 for La and Ce respectively, indicating that point defect interactions have a smaller effect than finite supercell size. The indirect method calculates the effective size misfits for the aliovalently doped systems by summing the size misfits of isolated neutral/charged point defects. This enables us to quantify the contribution of the point defect charge states to the effective size misfits.

Table 1: The size misfits $\left(\varepsilon_{b}\right)$ of single point defects in the neutral $\left(\operatorname{Ln}_{\mathrm{Sr}}\right.$ or $\left.V_{\mathrm{Sr}}\right)$ and the charged $\left(\operatorname{Ln}_{\mathrm{Sr}}^{\bullet}\right.$ or $\left.V_{\mathrm{Sr}}^{\prime \prime}\right)$ systems. The size misfit magnitudes and signs change when the point defects are charged: +1 for the substitutional atoms and -2 for the $\mathrm{Sr}$ vacancy.

\begin{tabular}{ccc}
\hline & $\varepsilon_{b}$ (neutral) & $\varepsilon_{b}$ (charged) \\
\hline $\mathrm{La}_{\mathrm{Sr}}$ & 0.0278 & -0.230 \\
$\mathrm{Ce}_{\mathrm{Sr}}$ & -0.0102 & -0.266 \\
$\mathrm{Nd}_{\mathrm{Sr}}$ & -0.0317 & -0.284 \\
$V_{\mathrm{Sr}}$ & -0.0652 & 0.317 \\
\hline
\end{tabular}

Table 1 shows the strong dependence of size misfits on the charge state of single point defects in $\mathrm{SrTiO}_{3}$. The negative size misfits for neutral Ce, Nd, and 
Figure 1: (color online). Geometry of $\mathrm{SrTiO}_{3}$ (left) with two substitutional atoms $\left(\mathrm{Ln}_{\mathrm{Sr}}=\right.$ $\mathrm{La}, \mathrm{Ce}$, and $\mathrm{Nd})$ and a $\mathrm{Sr}$ vacancy $\left(V_{\mathrm{Sr}}\right)$, and (right) with a single substitutional atom $\left(\mathrm{Ln}_{\mathrm{Sr}}\right)$ or a $\mathrm{Sr}$ vacancy $\left(V_{\mathrm{Sr}}\right)$. The supercells without defects contain $27 \mathrm{Sr}$ atoms (green), $27 \mathrm{Ti}$ atoms (blue), and $81 \mathrm{O}$ atoms (black). 
vacancy indicate that the cell volume contracts when these defects are present in $\mathrm{SrTiO}_{3}$, while La causes the cell to expand. When the substitutional atoms are in a +1 charge state (i.e. addition of a hole) and the vacancy is in a -2 charge state (i.e. addition of two electrons), the magnitudes of the size misfits change by more than a factor of four. The addition of a hole for the substitutional atoms decreases the size misfits, whereas the addition of electrons for the $\mathrm{Sr}$ vacancy increase the size misfit from its value for the neutral state. This strong dependence of size misfits of isolated single point defects on the charge state causes the effective size misfit obtained through the indirect method to also depend on the charge state.

Table 2: Comparison of effective size misfits $\left(\varepsilon_{b}\right)$ estimated from three different methods, followed by the size misfits difference (Diff.) which is an absolute error with respect to the direct method. The size misfits of point defects in aliovalently doped systems are directly computed from defective $\mathrm{SrTiO}_{3}$ containing two substitutional atoms and a Sr vacancy, or indirectly by linearly combining the size misfits of the neutral/charged single point defects. Accounting for the charge states of the defects in indirect method decreases the size misfit difference with respect to direct method less than 10\%.]]

\begin{tabular}{cccccc}
\hline & Direct Method & \multicolumn{2}{c}{ Indirect method, Neutral } & \multicolumn{2}{c}{ Indirect method, Charged } \\
& $\varepsilon_{b}$ & $2 \varepsilon_{b}\left(\operatorname{Ln}_{\mathrm{Sr}}\right)+\varepsilon_{b}\left(V_{\mathrm{Sr}}\right)$ & Diff. & $2 \varepsilon_{b}\left(\operatorname{Ln}_{\mathrm{Sr}}\right)+\varepsilon_{b}\left(V_{\mathrm{Sr}}^{\prime \prime}\right)$ & Diff. \\
\hline $\mathrm{La}_{\mathrm{Sr}}$ & -0.131 & -0.010 & -0.121 & -0.143 & 0.012 \\
$\mathrm{Ce}_{\mathrm{Sr}}$ & -0.199 & -0.086 & -0.113 & -0.215 & 0.016 \\
$\mathrm{Nd}_{\mathrm{Sr}}$ & -0.237 & -0.129 & -0.108 & -0.251 & 0.014 \\
\hline
\end{tabular}

Table 2 shows that accounting for the charge states of isolated point defects reduces differences in the effective size misfits of point defects in aliovalently doped $\mathrm{SrTiO}_{3}$ with respect to the direct method. The size misfits calculated using the direct method indicate that doping $\mathrm{SrTiO}_{3}$ with different substitutional atoms contracts the lattice by different amounts. The effective size misfits computed indirectly from the size misfits of neutral single point defects have large differences compared to the values obtained using the direct method, while the 
Figure 2: (color online). The size misfits $\left(\varepsilon_{b}\right)$ of the substitutional atoms $\left(\mathrm{Ln}_{\mathrm{Sr}}\right)$ and a vacancy $\left(V_{\mathrm{Sr}}\right)$ at a $\mathrm{Sr}$ site for different numbers of added/subtracted electrons. The size misfits vary linearly with similar slopes for substitutional atoms, whereas the linear variation for the $\mathrm{Sr}$ vacancy has a smaller slope. The slopes for $\mathrm{La}, \mathrm{Ce}, \mathrm{Nd}$, and a vacancy are $0.258,0.257,0.255$, and 0.190 , respectively.

indirect method applied to charged single point defects reduces the differences by large amount. We note that the size misfit from indirect method by accounting for the charge state of single point defects agrees with the size misfit from direct method to within $10 \%$ without charge correction. The remaining size misfit differences are attributed to the different dependence of size misfit between the substitutional atoms and the vacancy on extra charge state of point defects.

The size misfits of charged point defects depend linearly on their charge state [13, and Figure 2 shows that the sizes of an extra electron and a hole are dependent on the defect type in $\mathrm{SrTiO}_{3}$. We interpret the size misfit change due to charge state of the defective system as the effective size of an extra electron or a hole. The linear change of size misfits with the addition of an electron or a hole indicates that the hole size is the negative of the electron size for each defect. However, the magnitude of the electron/hole size depends on point defect type. Thus, the size of the two electrons in the Sr vacancy does not cancel the size of the two holes for two substitutional defects. The different dependence of the 
effective size of extra electron and hole on defect type is reflected in the charge distributions around the point defects in the neutral and charged systems.

\section{Charge distribution}

Figure 3 shows the difference in the charge distributions between two neutral single defects and aliovalently doped $\mathrm{SrTiO}_{3}$ compared to defect-free $\mathrm{SrTiO}_{3}$. When La substitutes for a Sr atom, electrons localize around the La atom and more charge transfers to $\mathrm{Ti}$ atoms than other elements. When there is a $\mathrm{Sr}$ vacancy, the holes localize around the $\mathrm{O}$ atoms near the vacancy. For the aliovalently doped system, charge transfer occurs due to the presence of multiple defects within the system resulting in the smaller charge localization around the defects comparing to the single defective systems. The localized electron charges of the single neutral La substitutional atom and a neutral Sr vacancy (i.e. the sum of the electron charge of the nearest $\mathrm{O}$ atoms) with respect to the undefective cell are 0.51 and -0.75 . Within the aliovalently doped system, the localized charges of each La atom and a Sr vacancy with respect to the undefective cell are 0.48 and -0.59 . The redistribution of charge in aliovalently doped $\mathrm{SrTiO}_{3}$ causes the different size misfits with respect to the indirect method applied on the neutral single defects.

Figure 4 shows that the extra hole and electrons introduced for charged single point defects are distributed throughout the supercell largely based on chemistry, not the distance from the defect. The extra hole for a La substitutional atom is distributed uniformly based on chemistry, with more transfer to Ti atoms than $\mathrm{Sr}$, La, or O. For a Sr vacancy, the two extra electrons are mostly distributed on the $\mathrm{O}$ atoms and rest of the extra electrons in between zero to average value of extra electrons (i.e. 2/135) distribute on the other atoms, but there are variations of magnitude for each atoms based on distance from the defect. This results in a small change in charge localization for the substitutional atom $(-0.01)$ and a larger change for the Sr vacancy (0.23). The charged defects compensate the difference of charge transfer between the neutral single point 
Figure 3: (color online). The difference in electron charge $\left(\Delta n_{e}\right)$ at each atomic position between the neutral defective $\mathrm{SrTiO}_{3}$ systems and undefective $\mathrm{SrTiO}_{3}$, with distance measured from the defect. The supercells contain a single La substitutional atom (top), a single $\mathrm{Sr}$ vacancy (bottom), and aliovalently doped $\mathrm{SrTiO}_{3}$ with two La substitutional atoms and a $\mathrm{Sr}$ vacancy (middle). The distance is measured from the La atom (top) or the Sr vacancy position (bottom, middle). The Sr vacancy position is estimated by averaging the positions of its 12 oxygen nearest neighbors in the relaxed cell. The distribution of electron charge at each atomic position is calculated using Bader charge integration. The charge transfer occurs within multiple defects in aliovalently doped supercell, which explains the different magnitude of charge localization near point defects compared to the neutral isolated defect supercells. 
Figure 4: (color online). The difference in electron charge $\left(\Delta n_{e}\right)$ between the charged defective $\mathrm{SrTiO}_{3}$ system and the neutral defective system, with distance measured from the defect. The supercells contain a single La substitutional atom (top) and a single vacancy (bottom) at a Sr site. The distance from the La substitutional atom or Sr vacancy is measured the same way as in Figure 3 The dashed lines show zero and the average electron density differences: $-1 / 135$ for $\mathrm{La}_{\mathrm{Sr}}^{\bullet}$ and $2 / 135$ for $V_{\mathrm{Sr}}^{\prime \prime}$. Both of an extra hole for the charged La substitutional atom and two extra electrons for charged Sr vacancy are not localized around defects, while showing different variations in spatial distribution. 
defects and aliovalently doped system. The electron charges for the La substitutional atom and a Sr vacancy in the charged supercells are 0.50 and -0.52 , respectively, which are closer to the charge distribution of the aliovalently doped system. We observed the same trend for other substitutional atoms. A more accurate description of the charge distribution of the isolated defects produces better agreement with the size misfits of the complexes. Also, it suggests that the compensating background charge generated due to additional charge for charged point defect does not hinder the size misfit comparison between direct and indirect method.]]

Our work predicts the same trend of size misfits as experiment [9, with the largest value for $\mathrm{La}$ and the smallest value for $\mathrm{Nd}$. The change of lattice parameter with $\mathrm{Sr}$ vacancy concentration is extracted from experimental data [9] to estimate the size misfits using Equation 2. The experimental size misfits of point defects in aliovalently doped $\mathrm{SrTiO}_{3}$ are $-0.023,-0.030$, and -0.151 for La, Ce and Nd substitutional atoms, respectively. Interestingly, this trend relates to the effective ionic radii of substitutional atoms $\mathrm{La}$, Ce and $\mathrm{Nd}$, which are 103, 101 and $98.3 \mathrm{pm}$ respectively [26]. The dopants with smaller ionic radii cause larger contraction of lattice, showing more negative value of size misfits for both of charged single substitutional atoms and aliovalently doped system. However, the magnitude of the experimental size misfits are smaller than what we find; it is possible this is due to multiple solute-solute, vacancy-vacancy and solute-vacancy interactions, as the experimental measurements include a much larger range of concentration than we consider here.

\section{Conclusion}

We describe a computationally efficient method to evaluate effective size misfits for aliovalently doped $\mathrm{SrTiO}_{3}$ that linearly combines the size misfits of charged single point defects. We find that using defect size misfits extracted from charged supercells matches well with the effective size misfits directly computed from the aliovalently doped system. This is explained by the different electron 
charge localization between the defective systems: the charge localization differences between the neutral single defects and the aliovalently doped system resulting from the charge transfer within multiple point defects, is compensated by the delocalized extra electron and hole distribution. We predict the same trend of size misfits as in experiments, the largest value for La and the smallest value for $\mathrm{Nd}$, while the absolute values are larger than experimental values. The linear combination of isolated defect calculations enables us to account for the charge state of each single defect, and faster finite-size convergence than for multiple defective geometries. We expect this method to efficiently estimate the effective size misfits for multiple defects with different charge states within $\mathrm{SrTiO}_{3}$ and other perovskite systems.

\section{Acknowledgments}

Figure 1 is drawn using the VESTA program [27]. This research was supported by NSF/MPS grant 10-52788. This work used the Extreme Science and Engineering Discovery Environment (XSEDE), which is supported by National Science Foundation grant number ACI-1053575 28].

\section{References}

[1] Kawai M, Watanabe S, Hanada T. Molecular beam epitaxy of $\mathrm{Bi}_{2} \mathrm{Sr}_{2} \mathrm{CuO}_{x}$ and $\mathrm{Bi}_{2} \mathrm{Sr}_{2} \mathrm{Ca}_{0.85} \mathrm{Sr}_{0.15} \mathrm{Cu}_{2} \mathrm{O}_{x}$ ultra thin films at $300{ }^{\circ} \mathrm{C}$. J Cryst Growth 1991;112(4):745 -52. URL: http://www.sciencedirect.com/ science/article/pii/002202489190131N doi/http://dx.doi.org/10. 1016/0022-0248(91)90131-N

[2] López-Suárez FE, Bueno-López A, Illán-Gómez MJ, Ura B, Trawczynski J. Potassium stability in soot combustion perovskite catalysts. Topics in Catalysis 2009;52(13-20):2097-100. URL: http://dx.doi.org/10.1007/ s11244-009-9385-z doi 10.1007/s11244-009-9385-z. 
[3] Haeni JH, Irvin P, Chang W, Uecker R, Reiche P, Li YL, et al. Room-temperature ferroelectricity in strained $\mathrm{SrTiO}_{3}$. Nature 2004;430:758-61. URL: http://www.nature.com/nature/journal/ v430/n7001/suppinfo/nature02773_S1.html. doi/http://dx.doi.org/ $10.1038 /$ nature02773

[4] Reaney IM, Colla EL, Setter N. Dielectric and structural characteristics of ba- and sr-based complex perovskites as a function of tolerance factor. Japanese Journal of Applied Physics 1994;33(7R):3984. URL: http:// stacks. iop.org/1347-4065/33/i=7R/a=3984.

[5] Lufaso MW, Woodward PM. Prediction of the crystal structures of perovskites using the software program SPuDS. Acta Crystallogr, Sect B: Struct Sci 2001;57(6):725-38. URL: http://dx.doi.org/10.1107/ S0108768101015282 doi $10.1107 /$ S0108768101015282.

[6] Zhang YM, Ubic R, Xue DF, Yang S. Predicting the structural stability and formability of $\mathrm{ABO}_{3}$-type perovskite compounds using artificial neural networks. Mater Focus 2012;1(1):57-64. URL: http://www . ingentaconnect. com/content/asp/mf/2012/00000001/00000001/art00008, doi:doi:10. $1166 /$ mat.2012.1009

[7] Giaquinta DM, Loye HCZ. Structural predictions in the $\mathrm{ABO}_{3}$ phase diagram. Chem Mater 1994;6(4):365-72. URL: http://dx.doi.org/10. 1021/cm00040a007, doi 10.1021/cm00040a007.

[8] Burn I, Neirman S. Dielectric properties of donor-doped polycrystalline $\mathrm{SrTiO}_{3}$. J Mater Sci 1982;17(12):3510-24. URL: http://dx.doi.org/10. 1007/BF00752196, doi $10.1007 / \mathrm{BF} 00752196$.

[9] Ubic R, Tolman K, Chan K, Lundy N, Letourneau S, Kriven WM. Effective size of vacancies in aliovalently doped $\mathrm{SrTiO}_{3}$ perovskites. J Alloys Compd 2013;575(0):239 -45. URL: http://www.sciencedirect.com/science/ article/pii/S0925838813010037. doi http://dx.doi.org/10.1016/j. jallcom.2013.04.091. 
[10] Varvenne C, Bruneval F, Marinica MC, Clouet E. Point defect modeling in materials: Coupling ab initio and elasticity approaches. Phys Rev B 2013;88:134102. URL: http://link.aps.org/doi/10.1103/PhysRevB. 88.134102. doi:10.1103/PhysRevB.88.134102.

[11] Kumagai Y, Oba F. Electrostatics-based finite-size corrections for firstprinciples point defect calculations. Phys Rev B 2014;89:195205. URL: http://link.aps.org/doi/10.1103/PhysRevB.89.195205 doi:10.1103/PhysRevB.89.195205

[12] Murphy ST, Hine NDM. Anisotropic charge screening and supercell size convergence of defect formation energies. Phys Rev B 2013;87:094111. URL: http://link.aps.org/doi/10.1103/PhysRevB. 87.094111. doi 10.1103/PhysRevB.87.094111.

[13] Bruneval F, Crocombette JP. Ab initio formation volume of charged defects. Phys Rev B 2012;86:140103. URL: http://link.aps.org/doi/10. 1103/PhysRevB.86.140103, doi 10.1103/PhysRevB.86.140103.

[14] Janotti A, Varley JB, Choi M, Van de Walle CG. Vacancies and small polarons in $\mathrm{SrTiO}_{3}$. Phys Rev B 2014;90:085202. URL: http://link.aps.org/doi/10.1103/PhysRevB.90.085202 doi $10.1103 /$ PhysRevB.90.085202.

[15] Middleburgh SC, Karatchevtseva I, Kennedy BJ, Burr PA, Zhang Z, Reynolds E, et al. Perozide defect formation in zirconate perovskites. J Mater Chem 2014;A:15883. URL: http://pubs.rsc.org/en/content/ articlehtml/2014/ta/c4ta02558j, doi 10.1039/C4TA02558J.

[16] Shanthi N, Sarma DD. Electronic structure of electron doped $\mathrm{SrTiO}_{3}: \mathrm{SrTiO}_{3-\delta}$ and $\mathrm{Sr}_{1-x} \mathrm{La}_{x} \mathrm{TiO}_{3}$. Phys Rev B 1998;57:2153-8. URL: http://link.aps.org/doi/10.1103/PhysRevB.57.2153. doi 10 . 1103/PhysRevB.57.2153. 
[17] Kresse G, Hafner J. Ab initio molecular dynamics for liquid metals. Phys Rev B 1993;47:558-61. URL: http://link.aps.org/doi/10.1103/ PhysRevB.47.558, doi:10.1103/PhysRevB.47.558.

[18] Kresse G, Furthmüller J. Efficiency of ab-initio total energy calculations for metals and semiconductors using a plane-wave basis set. Comp Mater Sci 1996;6(1):15 - 50. URL: http://www.sciencedirect.com/ science/article/pii/0927025696000080 doi/http://dx.doi.org/10. 1016/0927-0256(96)00008-0.

[19] Kresse G, Joubert D. From ultrasoft pseudopotentials to the projector augmented-wave method. Phys Rev B 1999;59:1758-75. URL: http://link.aps.org/doi/10.1103/PhysRevB.59.1758, doi 10 . 1103/PhysRevB.59.1758.

[20] Perdew JP, Burke K, Ernzerhof M. Generalized gradient approximation made simple. Phys Rev Lett 1996;77:3865-8. URL: http://link.aps.org/doi/10.1103/PhysRevLett.77.3865 doi $10.1103 /$ PhysRevLett.77.3865.

[21] Monkhorst HJ, Pack JD. Special points for brillouin-zone integrations. Phys Rev B 1976;13:5188-92. URL:/http://link.aps.org/doi/10.1103/ PhysRevB.13.5188, doi:10.1103/PhysRevB.13.5188.

[22] Bell RO, Rupprecht G. Elastic constants of strontium titanate. Phys Rev 1963;129:90-4. URL: http://link.aps.org/doi/10.1103/PhysRev.129. 90. doi $10.1103 /$ PhysRev.129.90.

[23] Piskunov S, Heifets E, Eglitis R, Borstel G. Bulk properties and electronic structure of $\mathrm{SrTiO}_{3}, \mathrm{BaTiO}_{3}, \mathrm{PbTiO}_{3}$ perovskites: an ab initio HF/DFT study. Comp Mater Sci 2004;29(2):165 -78. URL: http: //www.sciencedirect.com/science/article/pii/S0927025603001812. doi http://dx.doi.org/10.1016/j.commatsci.2003.08.036. 
[24] Yu M, Trinkle DR. Accurate and efficient algorithm for Bader charge integration. J Chem Phys 2011;134(6):064111. URL: http://scitation. aip.org/content/aip/journal/jcp/134/6/10.1063/1.3553716 doi:http://dx.doi.org/10.1063/1.3553716

[25] Yasi JA, Jr. LGH, Trinkle DR. First-principles data for solidsolution strengthening of magnesium: From geometry and chemistry to properties. Acta Mater 2010;58(17):5704 -13. URL: http: //www.sciencedirect.com/science/article/pii/S1359645410004106. doi:http://dx.doi.org/10.1016/j.actamat.2010.06.045.

[26] Shannon RD. Revised effective ionic radii and systematic studies of interatomic distances in halides and chalcogenides. Acta Crystallographica Section A 1976;32(5):751-67. URL: http://dx.doi.org/10.1107/ S0567739476001551, doi $10.1107 /$ S0567739476001551.

[27] Momma K, Izumi F. VESTA: a three-dimensional visualization system for electronic and structural analysis. Journal of Applied Crystallography 2008;41(3):653-8. URL: http://dx.doi.org/10.1107/ S0021889808012016, doi $10.1107 /$ S0021889808012016.

[28] Towns J, Cockerill T, Dahan M, Foster I, Gaither K, Grimshaw A, et al. Xsede: Accelerating scientific discovery. Computing in Science Engineering 2014;16(5):62-74. doi 10.1109/MCSE. 2014.80 


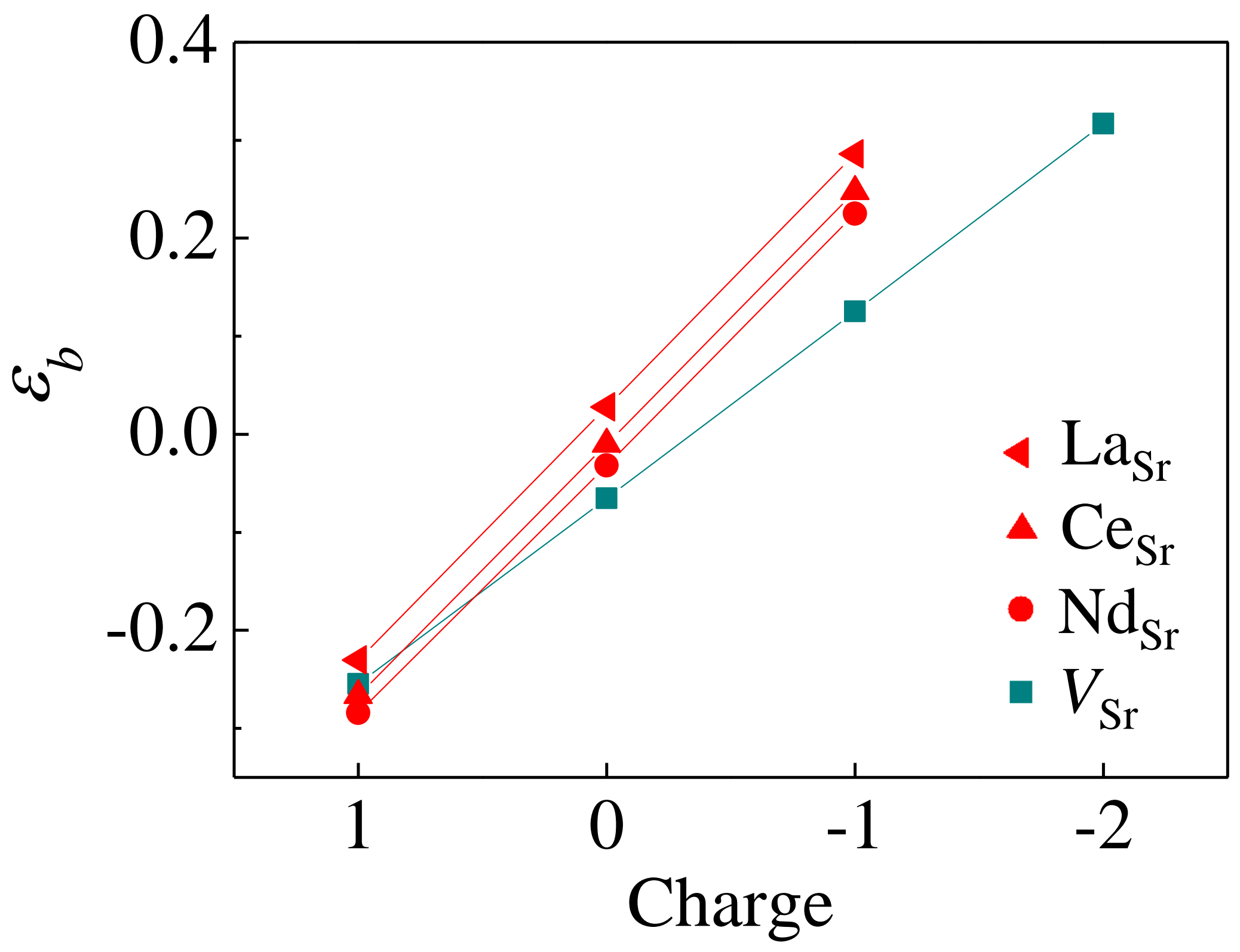

